\title{
Protocol
}

\section{Validation of generalized anxiety disorder-2 as a screening tool for generalized anxiety disorder in the primary health care setting: study protocol}

\author{
Mohammad J. M. A. Tamimi, Anna R. Sharif, Saad T. Sedeeq, Ehab Hamed* \\ Qatar University Health Center, Primary Health Care Corporation, Qatar \\ Received: 18 March 2021 \\ Revised: 18 April 2021 \\ Accepted: 19 April 2021 \\ *Correspondence: \\ Dr. Ehab Hamed, \\ E-mail: eshamed@gmail.com \\ Copyright: $\odot$ the author(s), publisher and licensee Medip Academy. This is an open-access article distributed under \\ the terms of the Creative Commons Attribution Non-Commercial License, which permits unrestricted non-commercial \\ use, distribution, and reproduction in any medium, provided the original work is properly cited.
}

\begin{abstract}
Background: Anxiety screening in primary care is crucial for the identification and management of the condition. The generalized anxiety disorder-2 (GAD-2) is an abridged version of the tool composed of the first two questions of the GAD-7 for screening for anxiety in the general adult population in primary care settings. Patients who score more than 3 points are further evaluated for anxiety. Although there is a growing body of literature documenting the psychometric properties of the GAD-2 against a structured interview and the GAD-7, no earlier studies have assessed the performance of the GAD-2 in Qatar. This cross-sectional study primarily aims to examine the sensitivity, specificity, positive (PPV) and negative predictive values (NPV) of GAD-2 scores for detecting anxiety among patients attending PHCC in Qatar. Methods: The study will employ a periodic cross-sectional study of electronic medical record data of adult patients attending the primary health care settings between January of 2017 and December of 2019. The study population will include all adult population with documented GAD-7 scores. We will exclude all patients with other mental health diagnoses, including mental disability, schizophrenia, and dementia.

Conclusions: GAD-2 questionnaire forms an easy to administer screening tool. The cut-off value in various international settings is set at a score of equal to or more than 3 . The study will report on the diagnostic properties of GAD-2 scores against the documented diagnosis of anxiety by health care professionals. The result helps to tailor the cut-off value to its local settings.
\end{abstract}

Trial Registration: Trial registration number is PHCCDCR202003019

Keywords: Generalized anxiety disorder, Psychometric properties, Health care professionals

\section{INTRODUCTION}

Mental disorders are widely recognized as a significant contributor $(14 \%)$ to the global burden of chronic disease worldwide. ${ }^{1}$ Patients often present to their family physician as their first contact with health services. Depression and anxiety disorders are the most common in such setups. Nevertheless, symptoms are commonly undetected. ${ }^{2}$ Lack of awareness and prominent levels of stigma related to mental health are barriers to accessing mental health services at an early stage, resulting in impairments in social functioning, employment, and less quality of life. Routine screening in primary care settings aids early detection and treatment and by turn could improve disease burden.

Generalized anxiety disorder (GAD) is one of the most common anxiety disorders in primary health care settings. The condition is characterized by persistent, excessive, and uncontrollable worry about everyday events or activities. ${ }^{3}$ Symptoms include fatigue, difficulty concentrating, sleep trouble, irritability, restlessness, and 
muscle tension. ${ }^{4}$ The chronic form of the disease is associated with suicidality, significant distress, and disability. Its manifestation is complicated by high comorbidity rates with other psychiatric disorders, such as major depressive disorder, panic disorder, and alcohol and substance abuse disorder, all of which aggravate outcomes and contribute to inadequate treatment response. ${ }^{5}$

The lifetime prevalence of GAD was estimated to be between $2.8 \%$ and $6.2 \%$, and the 12 -month prevalence rate was estimated at $0.9 \%$ to $2.9 \% .^{6}$ In the United States, the lifetime prevalence of anxiety disorders among adults aged 18 to 54 is $13.3 \% .^{7}$ Women are more susceptible to anxiety compared to men in most countries. ${ }^{9}$ Generalized anxiety disorder leads to considerable morbidity and affects 3 per cent of patients in primary care practices. ${ }^{10}$ In the primary care setting, the high prevalence of anxiety and depressive disorders increases the use of health services and delays response to treatments. ${ }^{7,8}$

Most people with anxiety disorders will be seen in primary care. ${ }^{11}$ Despite this, observational studies indicate that anxiety disorders are underdiagnosed and undertreated in this setting. ${ }^{12,13}$ Recently, it has been suggested that care might be improved if clinicians used standardized questionnaires to identify patients with anxiety disorders who have not previously been recognized. ${ }^{14}$

The generalized anxiety disorder-7 (GAD-7) and generalized anxiety disorder-2 (GAD-2) were designed for use in primary care patients. ${ }^{15,16}$ The GAD-7 consists of a self-report questionnaire; the 7 items questionnaire allows for the rapid detection of anxiety. ${ }^{15}$ Subjects are asked if they were bothered by anxiety-related problems over the past two weeks by answering a 4-point scale for each of the 7 items. The total scores ranged from 0 to 21 . Globally, among clinical and general population samples, the GAD7 has demonstrated good reliability and cross-cultural validity as a measure of GAD. ${ }^{17}$

The GAD-7 was developed in the USA as a valuable screening tool for detecting GAD in primary care patients. ${ }^{15}$ Pooled sensitivity and specificity values appeared acceptable at a cut-off point of 8 [sensitivity: 0.83 (95\% CI 0.71-0.91), specificity: 0.84 (95\% CI 0.70 0.92)]. The GAD-2 is an abridged version of the tool that is composed of the first two questions of the GAD-7 with a maximum score of $6 .{ }^{16} \mathrm{GAD}-2$ score is recommended for use for screening for anxiety in the general adult population in primary care settings. Both tests are widely applied by general practitioners in other primary care and family medicine settings. ${ }^{16}$

Current primary care guidelines in Qatar recommend the use of GAD-2 as a screening tool for all patients attending a primary health care setting. Patients who score more than 3 points are then asked to answer the complete assessment form, GAD-7, and then they have an interview with their family medicine doctor. Patients with a confirmed diagnosis have the diagnosis documented and start treatment according to local guidelines. Although there is a growing body of literature documenting the psychometric properties of the GAD-2 against a structured interview and the GAD-7, no previous studies have assessed the performance of the PHQ-2 in Qatar, AIMS.

This cross-sectional study aims to: examine the sensitivity, specificity, positive predictive values (PPV) and negative predictive values (NPV) of GAD-2 scores for detecting anxiety among patients attending PHCC in Qatar; report on the prevalence of anxiety using documented diagnosis by physicians and comparing that to prevalence for patients with a score of 8 or more on the GAD-7 as the criterion measure; and investigate the optimal cut-off score of the GAD-2 for screening and identifying cases of anxiety using in primary care settings.

\section{METHODS}

\section{Study design and settings}

The study will employ a periodic cross-sectional study of electronic medical record data of adult patients attending the primary health care settings between January of 2017 and December of 2019. Primary Health Care Corporation is a governmentally funded primary health care provider in Qatar and operates 27 health centres.

\section{Inclusion criteria}

We will include all adult population with documented GAD-7 scores. GAD-7 scores are done and documented in the notes by family medicine physicians if the physician feels an anxiety assessment is required. The age group include all adult population with age greater than or equal to 18 years old and younger than 65 years.

\section{Exclusion criteria}

We will exclude all patients with other mental health diagnoses, including mental disability, schizophrenia, and dementia, e.g. no sampling calculation is required as all population will be included.

\section{Data management}

The study will utilize anonymous data collection and analysis and will ensure compliance with research ethical and quality requirements. Only LPI and data analyst will have access to the anonymized data set. All investigators will follow the requirements of the general data protection regulations and data protection requirements regarding the collection, storage, processing of the data according to institutional protocols.

Variables include: GAD-2 scores and dates; GAD-7 scores and dates; non-identifiable demographics including age in years, nationality, relationship status, gender; comorbid conditions (diabetes mellitus (yes or no), hypertension (yes or no), dyslipidaemia (yes or no), hypothyroidism; and 
mental health diagnosis, including anxiety, depression, schizophrenia, dementia, etc.

\section{Statistical analysis and research outcomes}

Statistical analysis will include all population eligible for the study and no sample size calculation would be needed. Statistical outcomes will include: a descriptive analysis of population demographics, GAD-2 score, GAD-7 scores, and anxiety as a diagnosis; the diagnostic characteristics and the discriminative validity of the GAD-2 questionnaire against the documented diagnosis of anxiety by reporting on sensitivity, specificity, and Youden index; and the optimal cut-off point for use as a screening tool in local settings by comparing all scores from 0-6.

\section{Study registration}

The primary health care corporation research committee has approved the study. The research committee approval reference number is PHCCDCR202003019.

\section{DISCUSSION}

Anxiety and depression are the most common mental health disease presentations in primary health care settings. ${ }^{3}$ Anxiety prevalence among adults over 12 months is reported as high as $3 \% .^{3}$

Albeit the condition is underdiagnosed due to stigma and lack of recognition in many international settings. Screening tools aim to detect undiagnosed cases and aids early interventions.

GAD-2 questionnaire forms an easy to administer screening tool in primary health care settings. The cut-off value in various international settings is set at a score of equal to or more than $3 .{ }^{16}$ Patients are then recommended to have a full assessment. While that cut-off value has been assessed in various settings, national studies should focus on investigating the diagnostic characteristics in such tools in various settings. The current recommended cut-off value of 3 or more may under or overdiagnoses the condition in different populations or settings.

We aim to investigate GAD-2 questionnaire as a screening tool for anxiety in the primary care settings of Qatar. Guidelines' developers and policymakers should consider the local population variance and studies that are specific to local settings. Reporting on the diagnostic characteristics of the tool among the local population is essential to decide the population-specific cut-off value. Similarly, researchers should prioritize investigations of the cut-off values of internationally developed tools in their settings. Comparing the performance of the tool in different international settings would help our understanding and development of the tool. Research of such domains is crucial for the early detection of the condition and decreasing disease burden.

\section{CONCLUSION}

Anxiety is a common mental health presentation in primary health care settings. Tools may help to identify early presentations. Using the internationally validated tool is highly recommended, yet examining the cut-off point is crucial to attaining the same result. Researchers should ideally aim to develop tools for local settings or report on the diagnostic properties of the tool. Policymakers and guidelines developers should consider those when deciding on adopting internationally developed tools to their local guidance.

\section{ACKNOWLEDGEMENTS}

Authors would like to acknowledge the support they received from the Primary healthcare corporation research department.

\section{Funding: No funding sources \\ Conflict of interest: None declared \\ Ethical approval: The study was approved by the Institutional Ethics Committee}

\section{REFERENCES}

1. Prince M, Patel V, Saxena S, Maj M, Maselko J, Phillips MR, Rahman A. No health without mental health. Lancet. 2007;370(9590):859-77.

2. Mechanic D. Barriers to help-seeking, detection, and adequate treatment for anxiety and mood disorders: Implications for health care policy. J Clin Psychiatr. 2007;68:20-6.

3. Newman MG, Llera SJ, Erickson TM, Przeworski A, Castonguay LG. Worry and generalized anxiety disorder: a review and theoretical synthesis of evidence on nature, etiology, mechanisms, and treatment. Annu Rev Clin Psychol. 2013;9:275-97.

4. Diagnostic and Statistical Manual of Mental Disorders [DSM]. 4th ed. Text Revision; American Psychiatric Association. 2004.

5. Maron E, Nutt D. Biological markers of generalized anxiety disorder. Dialogues Clin Neurosci. 2017;19(2):147-58.

6. Bandelow B, Michaelis S. Epidemiology of anxiety disorders in the 21st century. Dialogues Clin Neurosci. 2015;17(3):327-35.

7. Ghuloum S, Bener A, Burgut FT. Epidemiological survey of knowledge, attitudes, and health literacy concerning mental illness in a national community sample: a global burden. J Prim Care Comm Health. 2010;1:111-8.

8. Bener A, Guloum S. Gender differences in the knowledge, attitude and practice towards mental health illness in a rapidly developing Arab society. Int J Soc Psychiatr. 2011;57:480-6.

9. Gigantesco A, Palumbo G, Mirabella F, Pettinelli M, Morosini P. Prevalence of psychiatric disorders in an Italian Town: Low prevalence confirmed with two 
different interviews. Psychother Psychosom. 2006;75:170-6.

10. Leon AC, Olfson M, Broadhead WE, et al. prevalence of mental disorders in primary care. Implications for screening. Arch Fam Med. 1995;4(10):857-61.

11. Lieb R, Becker E, Altamura C. The epidemiology of generalized anxiety disorder in Europe. Eur Neuropsychopharmacol. 2005;15:445-52.

12. Fernandez A, Rubio-Valera M, Bellon JA, PintoMeza A, Luciano JV, Mendive JM, et al. recognition of anxiety disorders by the general practitioner: results from the DASMAP Study. Gen Hosp Psychiatr. 2012;34:227-33.

13. Stein MB, Sherbourne CD, Craske MG, MeansChristensen A, Bystritsky A, Katon W, et al. quality of care for primary care patients with anxiety disorders. Am J Psychiatr. 2004;161:2230-7.

14. Katon W, Roy-Byrne P. Anxiety disorders: efficient screening is the first step in improving outcomes. Ann Intern Med. 2007;146:390-1.
15. Spitzer RL, Kroenke K, Williams JB, Löwe B. A brief measure for assessing generalized anxiety disorder: the GAD-7. Arch Intern Med. 2006;166:1092-7.

16. Kroenke K, Spitzer RL, Williams JB, Monahan PO, Löwe B. Anxiety disorders in primary care: prevalence, impairment, comorbidity, and detection. Ann Intern Med. 2007;146:317-25.

17. Löwe B, Decker O, Müller S, Brähler E, Schellberg $\mathrm{D}$, Wolfgang $\mathrm{H}$, et al. Validation and standardization of the Generalized Anxiety Disorder Screener (GAD7 ) in the general population. Med Care. 2008;46.

Cite this article as: Tamimi MJMA, Sharif AR, Sedeeq ST, Hamed E. Validation of generalized anxiety disorder-2 as a screening tool for generalized anxiety disorder in the primary health care setting: study protocol. Int J Clin Trials 2021;8(2):152-5. 